\title{
The Application Value of the Follow-up Platform of Breast Specialty Combined with Individualized Therapy Book in Breast Cancer Patients
}

\author{
Wan Tang, Yuhua Ma*, Xiuli Wei \\ General Surgery Department, The First Affiliated Hospital of Jinan University, Guangzhou, China \\ Email address: \\ 819047293@qq.com (Wan Tang),924006463@qq.com (Yuhua Ma),445827039@qq.com (Xiuli Wei) \\ ${ }^{*}$ Corresponding author
}

To cite this article:

Wan Tang, Yuhua Ma, Xiuli Wei. The Application Value of the Follow-up Platform of Breast Specialty Combined with Individualized Therapy Book in Breast Cancer Patients. American Journal of Nursing Science. Vol. 9, No. 4, 2020, pp. 200-203. doi: 10.11648/j.ajns.20200904.15

Received: May 3, 2020; Accepted: May 22, 2020; Published: June 3, 2020

\begin{abstract}
Objective: To assess the application value of the follow-up platform of breast specialty combined with individualized therapy book in breast cancer patients. Methods: in our study, total participants have 180 who are diagnosed as breast cancer from November 2018 to December 2019, the control group has 90 participants who provide their reports in follow-up, and the intervention group has 90 participants, they provide their data in follow-up. The control group participants receive conventional follow-up. The intervention group participants receive the follow-up platform and individualized therapy book. Our researchers collect the data by interview and questionnaires, the data include compliance assessment, range of motion of shoulder after operation, patient satisfaction, relevant knowledge of patient assessment, life quality, anxiety assessment, and depression assessment. The questionnaires of our study follow as: Function Assessment of Cancer Therapy-Breast (FACT-B), Self-Rating Anxiety Scale (SAS), Self-rating depression scale (SDS), and other simple questionnaires. Result: The characteristics of two group are similar as the analysis results are not statistical significance $(p=0.091, p=0.757, p=0.425 p=$ 0.326). The range of motion of the shoulder research indicates the range of motion of the shoulder in follow-up. In particular, intervention group participants have more good range of motion of the shoulder in our research than that of control group. In patient satisfaction, most intervention group participants provide 'very well' assessment in satisfaction research, it has more those assessments than that of control group $(\mathrm{n}=81 \mathrm{vs} \mathrm{n}=54)$. Conclusion: The follow-up platform and the individualized therapy book improve the patient recovery rate, patient compliance, patient satisfaction, quality of life, mental health, and their degree of related information mastery after related operation of breast cancer.
\end{abstract}

Keywords: Breast Cancer, Follow-up Platform, Individualized Therapy Book

\section{Introduction}

In all health problems, the breast cancer is a common problem in worldwide, it had ranks tops which are estimated new cancer cases and deaths in all types of cancer [1, 2]. Base on the report, breast cancer patient had a small population of cells that display stem cell properties, that contain self-renewal and clonal long-term repopulation capacity [3]. These breast cancer stem cells (BCSCs) are responsible for radiation and chemotherapy resistance. Even a rare breast cancer stem cells population can give rise to tumors [4]. In addition, BCSCs play an important role during the initiation, proliferation, invasion and relapse of breast cancer [5].
Compared with other cancers, breast cancer is happened in the place which has lots of fat pad, the fat pad providing the basic niche for tumor initiation and progression. Therefore, strategies aimed at targeting this cell population may be a more effective therapies for breast cancer [6]. Moreover, high levels of fatty acid will provide tumor cells with lipid material to facilitate tumor growth and maintain BCSCs properties.

The rapid development of high-throughput technologies and computational frameworks of last decades allowed to study the biological systems in unprecedented detail and at different omics levels leading in turn to significant advances in precision medicine [7]. Rarely a drug is $100 \%$ effective and safe and some therapies succeed only to small groups of 
patients affected with the same disease. Therefore, the patients receive the precision treatment by enough patient information [8]. it involves the ability to classify individuals into subpopulations that are uniquely or particularly susceptible to a particular disease or responsive to a specific treatment. Aim of this study is that assess the application value of the follow-up platform of breast specialty combined with individualized therapy book in breast cancer patients.

\section{Methods}

\subsection{Participants Enrollment and Survey Methods}

This study invests 183 patients to participate in follow-up, who are diagnosed as breast cancer from November 2018 to December 2019. But 3 participants are excluded as we could not contact them. Therefore, in our study, total participants have 180, the control group has 90 participants who provide their reports in follow-up, and the intervention group has 90 participants, they provide their data in follow-up. The control group participants receive conventional follow-up. The intervention group participants receive the follow-up platform and individualized therapy book. Our researchers collect the data by interview and questionnaires, the data include compliance assessment, range of motion of shoulder after operation, patient satisfaction, relevant knowledge of patient assessment, life quality, anxiety assessment, and depression assessment. The questionnaires of our study follow as: Function Assessment of Cancer Therapy-Breast (FACT-B), Self-Rating Anxiety Scale (SAS), Self-rating depression scale (SDS), and other simple questionnaires [9-11].

the follow-up platform is built from hospital, it is order to collect the information of patient in follow-up. Our researchers get the related information from this follow-up platform. In addition, the individualized therapy book records the individualized treatment method and patient situation, it is order to improve the influence of treatment for different people.

Their inclusion criteria were: (1) the patients were diagnosed as breast cancer; (2) voluntarily participate in the study and give informed consent, without cognitive impairment, able to understand the content of health education and cooperate with the completion of follow-up; (3) patients and their families can skillfully use mobile phones and/or computers. Their withdraw criteria were: (1) complicated with heart, liver, kidney, brain and other vital organ diseases; (2) mental disorder or language communication disorder; (3) with tumor recurrence, metastasis or other malignant tumors; (4) recently used anti-anxiety and depression $\mathrm{d}$.

\subsection{Statistical Analysis}

Our data analyzer performed the statistical analysis by SPSS 22.0. The $\mathrm{P}$ value, t-test and chi-square test were associated with collection result were analyzed. Besides, the mean standard deviation for statistical description.

\section{Result}

The Table 1 shows the patient characteristics in this study, that contains patient age, operation method, breast cancer stage of patient and education Level of patient. The characteristics of two group are similar as the analysis results are not statistical significance $(p=0.091, p=0.757, p=$ $0.425 \mathrm{p}=0.326)$. In breast cancer stage, most participants are the second stage and the third stage. In addition, most participants are primary school and junior high school in education career.

Table 1. Patient Characteristics.

\begin{tabular}{llll}
\hline Projects & Intervention group $(\mathbf{n}=\mathbf{9 0})$ & Control group $(\mathbf{n}=\mathbf{9 0})$ & P value \\
\hline Age (year) & $54.87 \pm 12.14$ & $52.00 \pm 10.27$ & 0.091 \\
Operation method (modified) & 34 & 32 & 0.757 \\
Breast cancer stage & 5 & 8 \\
Phase I & 26 & 29 \\
Phase II & 38 & 40 \\
Phase III & 21 & 13 \\
Phase IV & & & 0.425 \\
Education Level & 39 & 32 \\
Primary school & 26 & 35 \\
Junior high school & 8 & 4 \\
High school & 17 & 19 \\
College degree or above & & 0.326 \\
\hline
\end{tabular}

In patient compliance research, the intervention group has better compliance than that of control group (Table 2). In different level of compliance, most participants provide 'complete' level. But their results are statistical significance ( $p$ $=0.001)$, those assessments are more in intervention group compare with control group.

Table 2. Patient Compliance.

\begin{tabular}{lllll}
\hline Projects & Complete & Sectional & Bad & Compliance rate (\%) \\
\hline Intervention group $(\mathrm{n}=90)$ & 60 & 24 & 6 & 93.3 \\
Control group $(\mathrm{n}=90)$ & 38 & 35 & 17 & 84.4 \\
P Value & 0.001 & 0.080 & 0.014 \\
\hline
\end{tabular}

In Table 3, it indicates the range of motion of the shoulder in 
more good range of motion of the shoulder in our research than that of control group.

Table 3. Range of motion of the shoulder.

\begin{tabular}{llll}
\hline Projects & Good & common & Bad \\
\hline Intervention group $(\mathrm{n}=90)$ & 30 & 38 & 22 \\
Control group $(\mathrm{n}=90)$ & 14 & 40 & 36 \\
P Value & 0.006 & 0.764 & 0.026 \\
\hline
\end{tabular}

In Table 4, it contains patient satisfaction and their degree of related information mastery. In patient satisfaction, most intervention group participants provide 'very well' assessment

in satisfaction research, it has more those assessments than that of control group $(n=81$ vs $n=54)$.

Table 4. Patient satisfaction and their degree of related information mastery.

\begin{tabular}{|c|c|c|c|c|c|c|c|}
\hline \multirow{2}{*}{ Projects } & \multicolumn{4}{|c|}{ Patient satisfaction } & \multicolumn{3}{|c|}{ degree of related information mastery } \\
\hline & Very well & Good & Pass & Bad & Good & Common & Bad \\
\hline Intervention group $(\mathrm{n}=90)$ & 81 & 6 & 3 & 0 & 78 & 10 & 2 \\
\hline Control group $(n=90)$ & 54 & 18 & 15 & 3 & 56 & 29 & 5 \\
\hline P Value & 0.000 & 0.009 & 0.003 & 0.244 & 0.001 & 0.005 & 0.441 \\
\hline
\end{tabular}

In quality of life research, the result contains 2 parts, such as result of just out of the hospital and result of three months after discharge. In 5 domains, the result of just out of the hospital is not statistical significance, but the result of three months after discharge is statistical significance (Table 5).

Table 5. Quality of life assessment (Mean $\pm S D)$.

\begin{tabular}{|c|c|c|c|c|c|c|c|c|c|c|}
\hline \multirow{2}{*}{ Projects } & \multicolumn{2}{|c|}{ Body Functions } & \multicolumn{2}{|c|}{ Role Functions } & \multicolumn{2}{|c|}{ Emotion Functions } & \multicolumn{2}{|c|}{ Social Functions } & \multicolumn{2}{|c|}{ Cognitive function } \\
\hline & $\mathbf{J P}$ & TM & JP & TM & JP & TM & JP & TM & $\mathbf{J P}$ & $\mathbf{T M}$ \\
\hline $\begin{array}{l}\text { Intervention } \\
\text { group }(n=90)\end{array}$ & $66.78 \pm 11.14$ & $79.12 \pm 9.31$ & $64.61 \pm 6.12$ & $73.71 \pm 5.35$ & $64.36 \pm 5.78$ & $75.28 \pm 5.19$ & $65.66 \pm 5.43$ & $75.23 \pm 5.14$ & $45.64 \pm 2.17$ & $57.37 \pm 1.26$ \\
\hline $\begin{array}{l}\text { Control group } \\
(\mathrm{n}=90)\end{array}$ & $67.82 \pm 10.94$ & $71.45 \pm 10.11$ & $65.31 \pm 5.88$ & $72.24 \pm 4.87$ & $64.95 \pm 6.42$ & $69.75 \pm 6.2$ & $64.47 \pm 5.12$ & $69.65 \pm 4.98$ & $45.54 \pm 2.38$ & $48.41 \pm 2.42$ \\
\hline P Value & 0.314 & $<0.005$ & 0.072 & 0.017 & 0.716 & $<0.005$ & 0.192 & $<0.005$ & 0.216 & 0.037 \\
\hline
\end{tabular}

$\mathrm{JP}=$ Just out of the hospital

$\mathrm{TM}=$ Three months after discharge

In Table 6, the intervention group has better performance than that of control group in SAS and SDS. In the period of just out of the hospital, the data of two group are similar as its result is not statistical significance. In addition, intervention group participants provide better assessment in three months after discharge, it is statistical significance.

Table 6. Result of anxiety assessment and depression assessment (Mean $\pm S D)$.

\begin{tabular}{|c|c|c|c|c|}
\hline \multirow{2}{*}{ Projects } & \multicolumn{2}{|l|}{ SAS } & \multicolumn{2}{|l|}{ SDS } \\
\hline & JP & TM & JP & TM \\
\hline Intervention group $(\mathrm{n}=90)$ & $57.4 \pm 7.2$ & $46.5 \pm 8.1$ & $58.9 \pm 6.8$ & $52.4 \pm 7.1$ \\
\hline Control group $(n=90)$ & $63.8 \pm 7.6$ & $57.2 \pm 7.4$ & $58.6 \pm 5.9$ & $54.6 \pm 6.2$ \\
\hline P Value & 0.171 & $<0.005$ & 0.214 & $<0.005$ \\
\hline
\end{tabular}

$\mathrm{JP}=$ Just out of the hospital

$\mathrm{TM}=$ Three months after discharge

\section{Discussion and Conclusion}

Deaths from breast cancer have progressively declined over the past twenty years [12]. Women can live longer who are diagnosed with breast cancer, but increased breast cancer survivorship presents physical and psychosocial challenges for survivors [13]. Cancer survivorship refers to the phase following treatment completion when women have to adapt to the new situation [14]. Base on the report, approximately 500,000 annual deaths are attributed to breast cancer worldwide, and 1 in every 8 to 10 women develop breast cancer [15]. The survival rate of patients with breast cancer in the developed and developing countries varies significantly. The 5-yearsurvival rate of patients with breast cancer in the developed countries can be as high as $80 \%$. In contrast, the 5 -year survival rate of patients with breast cancer in the developing countries is lower than $40 \%$ [16, 17]. Thus, the follow-up platform and the individualized therapy book are important for postoperative breast cancer patients, they provide more accurate patient status to researchers.

According to above result, the follow-up platform and the individualized therapy book improve the patient recovery rate, patient compliance, patient satisfaction, quality of life, mental health, and their degree of related information mastery after related operation of breast cancer. In patient compliance, 
easier receive treatment and recovery according to doctor asking in intervention group patient compare with control group. In addition, the follow-up platform and the individualized therapy book improve the patient recovery rate as intervention group has better the range of motion of the shoulder result than that of control group, Base on patient satisfaction and their degree of related information mastery, intervention group has higher assessment in those researches, it indicates the follow-up platform and the individualized therapy book can improve mental health of patient. In limitation, we did not make the professional mental health research, so we only infer the follow-up platform and the individualized therapy book can improve the mental health as better degree of related information mastery of patient can influence their mental health. However, we cannot ensure that it has strongest influence for which negative emotions.

\section{References}

[1] Bray F, Ferlay J, Soerjomataram I, Siegel RL, Torre LA, Jemal A. Global cancer statistics 2018: GLOBOCAN estimates of incidence and mortality world-wide for 36 cancers in 185 countries, CA Cancer J. Clin. 2018; 68 (6): 394-424.

[2] Siegel RL, Miller KD, Jemal A. Cancer statistics, 2018, CA Cancer J. Clin. 2018; 68 (1): 7-30.

[3] Liu S, Wicha MS. Targeting breast cancer stem cells, J. Clin. Oncol. 2016; 28 (25): 4006-4012.

[4] McDermott P, Wicha MS. Targeting breast cancer stem cells, Mol. Oncol. 2017; 4 (5): 404-419.

[5] Ginestier C, Charafe-Jauffret E, Birnbaum D. Targeting breast cancer stem cells: fishing season open!, Breast Cancer Res. 2015; 12 (5): 312.

[6] Wiseman BS, Werb Z. Stromal effects on mammary gland development and breast cancer, Science. 2018; 296 (5570): $1046-1049$.

[7] Mathur S, Sutton J. Personalized medicine could transform healthcare, Biomed. Rep. 2017; 7: 3-5.

[8] Ashley EA. Towards precision medicine, Nat. Rev. Genet. 2016; 17: 507-522.

[9] White D, Leach C, Sims R, Atkinson M, Cottrell D. Validation of the Hospital Anxiety and Depression Scale for use with adolescents. Br J Psychiatry 1999; 175: 452-454.

[10] El-Rufaie O, Absood G. Validity study of the Hospital Anxiety and Depression Scale among a group of Saudi patients. Br J Psychiatry 1987; 151: 687-688.

[11] Zonghua W, Dongmei Z, Xueliang T, et al. Revision of the Chinese Version of the FACT-B for Patients with Breast Cancer Chinese Mental Health Journal. 2006; (2): 184-187.

[12] Miller KD, Siegel RL, Lin CC, Mariotto AB, Kramer JL, Rowland JH, Stein KD, Alteri R, Jemal A. Cancer treatment and survivorship statistics, 2016; 66 (4): 271-289.

[13] Maher EJ, Fenlon DR. The psychosocial issues of survivorship in breast cancer, Adv Breast Cancer. 2015; 7 (2): 17-22.

[14] Cheng KKF, Lim YTE, Koh ZM, Tam WWS. Home-based multidimensional survivorship programmes for breast cancer survivors, Cochrane Database of Systematic Reviews. 2017; (8): 1-149.

[15] Tao A, Shi C, Lu, et al. Breast cancer: epidemiology and etiology, Cell Biochem. Biophys. 2015; 72: 333-338.

[16] Coleman P, Quaresma M, Berrino F, et al., Cancer survival in five continents: a worldwide population-based study (CONCORD), Lancet Oncol. 2008; 9: 730-756.

[17] Han J, Guo QQ, Wang T, et al., Prognostic significance of interactions betweenER alpha and ER beta and lymph node status in breast cancer cases, Asian Pac. J. Cancer Prev. APJCP: Asian Pac. J. Cancer Prev. APJCP. 2016; 14: 6081-6084. 